\title{
CD147 silencing inhibits tumor growth by suppressing glucose transport in melanoma
}

\author{
Juan Su' ${ }^{1,2}$, Tianyuan Gao ${ }^{1,3}$, Minghao Jiang ${ }^{1,4}$, Lisha Wu ${ }^{5}$, Weiqi Zeng ${ }^{1,2}$, Shuang \\ Zhao $^{1,2}$, Cong Peng ${ }^{1,2}$ and Xiang Chen ${ }^{1,2}$ \\ ${ }^{1}$ Department of Dermatology, Xiangya Hospital, Central South University, Changsha, Hunan, China \\ ${ }^{2}$ Hunan Key Laboratory of Skin Cancer and Psoriasis, Xiangya Hospital, Central South University, Changsha, Hunan, China \\ ${ }^{3}$ Department of Dermatology, the First Affiliated Hospital of Xi'an Jiaotong University, Xi'an, Shanxi \\ 4 Department of Anesthesiology, Xiangya Hospital, Central South University, Changsha, Hunan, China \\ ${ }^{5}$ Institute of Medical Science Research, Xiangya Hospital, Central South University, Hunan, China \\ Correspondence to: Xiang Chen, email: chenxiangck@126.com
}

Cong Peng, email: pengchongpeng@hotmail.com

Keywords: melanoma; CD147; glycolysis; GLUT-1; PI3K/Akt pathway

Received: June 29, $2016 \quad$ Accepted: August 15, 2016

Published: August 19, 2016

\section{ABSTRACT}

Melanoma is a very malignant disease and there are still no effective treatments. CD147 participates in the carcinogenesis of multiple human cancers and GLUT-1, as a glucose transporter, is associated with tumor growth. However, the function of CD147 and GLUT-1 in melanoma have not been completely understood. Thus, in this study we investigated the expression of CD147 and GLUT-1 in melanoma tissue, which were overexpressed compared with that in nevus tissue. In addition, CD147 and GLUT-1 were co-localized in the cytoplasm of human melanoma A375 cells. Immunoprecipitation proved that CD147 interacted with GLUT-1 at D105-199. Silencing CD147 by specific siRNA could downregulate GLUT-1 level via inhibiting PI3K/Akt signaling and decrease glucose uptake in A375 cells. In vivo experiments also supported that CD147 knockdown suppressed the tumor growth in melanoma subcutaneous mice model, observed by micro PET/CT. Our results could help validate CD147 as a new therapeutic target for treating melanoma.

\section{INTRODUCTION}

Melanoma is one of the highly malignant and progressive tumors, and the incidence and mortality have been on the increase year by year [1]. In the USA, melanoma was the sixth commonest malignant tumor [2]. Most patients with this deadly disease are associated with poor prognosis and the median survival was around 6 months. Light-colored skin or hair, photosensitivity, sunlight exposure and positive family history could increase the risk for melanoma [3]. However, the potential molecular mechanism for melanoma has not been completely understood, and there are still no effective treatments available.

Glycolysis could provide energy for the tumor growth. Recent studies have proved that the regulation of glycolysis might exert anti-tumor activities in multiple human cancers [4]. Glucose transporter (GLUT) family is kind of glucose transporter protein, which was overexpressed in tumor tissue [5]. GLUT-1 as one member of the GLUT family is a transmembrane glycoprotein located on cell membrane and mediates the transportation of glucose. It was reported that GLUT-1 was upregulated in melanoma and was correlated with the tumor differentiation [6]. In addition, the expression of GLUT-1 may predict the hypoxia and glycolysis in tumor tissue as well as the patients' outcome. Recently, CD147 has been shown to participate the development and progression of melanoma [7] and is associated with the malignant phenotype, but whether CD147 could regulate the glucose transport in melanoma is still unknown. Thus, in this study we investigated the effect of CD147 silencing on the glucose transport and its interaction with GLUT in melanoma. Our results may help expand the current understanding on the carcinogenesis of melanoma. 
Table 1: CD147 and GLUT-1 were overexpressed in melanoma.

\begin{tabular}{|c|c|c|c|c|c|c|}
\hline & \multicolumn{2}{|c|}{ Nevus $(\boldsymbol{n}=\mathbf{2 2})$} & \multicolumn{2}{c|}{ Melanoma $(\boldsymbol{n}=\mathbf{2 2})$} & \multirow{2}{*}{$\boldsymbol{X}^{\mathbf{2}}$} & \multirow{2}{*}{$\boldsymbol{P}$ value } \\
\hline & Low & High & Low & High & & 0.003 \\
\hline CD147 & 12 & 10 & 2 & 20 & 10.476 & 0.002 \\
\hline GLUT-1 & 15 & 7 & 4 & 18 & 11.208 & 0.1 \\
\hline
\end{tabular}

Table 2: High CD147 and GLUT-1 level was observed in metastatic melanoma compared with primary melanoma.

\begin{tabular}{|c|c|c|c|c|c|c|}
\hline & \multicolumn{2}{|c|}{ Primary melanoma $(\boldsymbol{n}=\mathbf{1 2 7})$} & Metastatic melanoma $(\boldsymbol{n}=\mathbf{6 2})$ & \multirow{2}{*}{$\mathbf{X}^{2}$} & \multirow{2}{*}{$\boldsymbol{P}$ value } \\
\cline { 1 - 5 } & Low & High & Low & High & & 0.006 \\
\hline CD147 & 45 & 82 & 10 & 52 & 7.525 & 0.036 \\
\hline
\end{tabular}

\section{RESULTS}

\section{CD147 was correlated with GLUT-1 in melanoma}

We first investigated the expression of CD147 and GLUT-1 in nevus $(n=20)$ and melanoma $(n=20)$ tissue. IHC analysis showed that both CD147 and GLUT-1 were

A

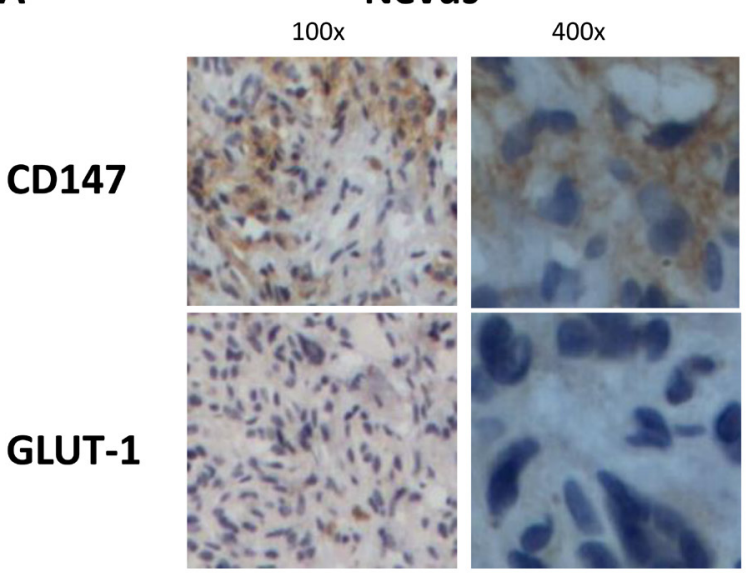

C

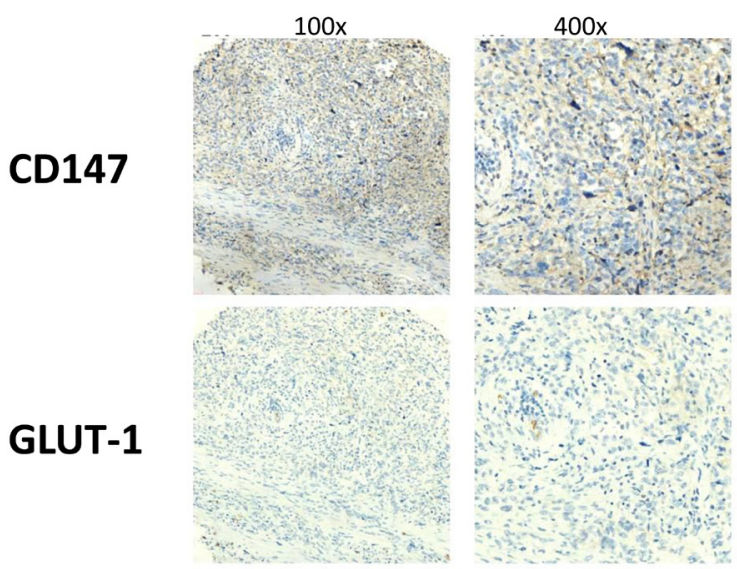

overexpressed in melanoma tissue compared with nevus tissue $(P=0.003$ for CD147, $P=0.002$ for GLUT-1; Table 1, Figure 1A-1B). Our results also supported that metastatic melanoma was associated with higher CD147 and GLUT-1 level than primary melanoma $(P=0.006$ for CD147, $P=0.036$ for GLUT-1; Table 2, Figure 1C1D), indicating that CD147 and GLUT-1 might play an important role in the development and progression of melanoma.

B

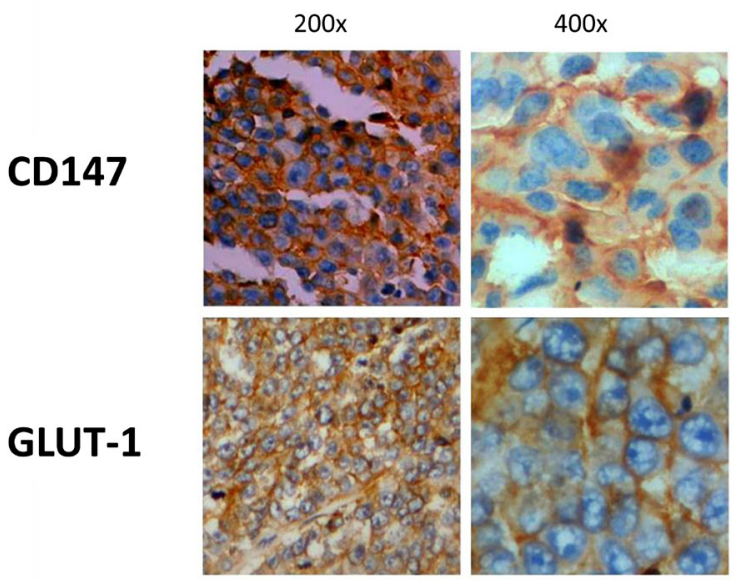

D

\section{Metastasis}

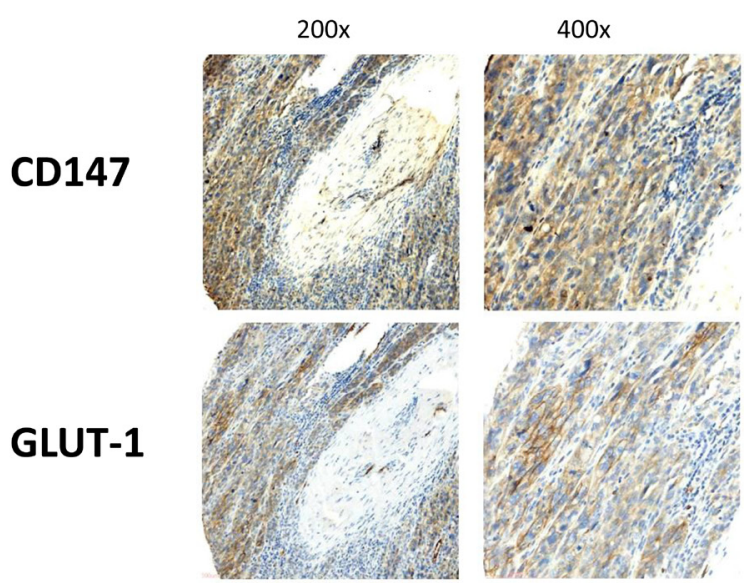

Figure 1: CD147 and GLUT-1 were overexpressed in melanoma compared with nevus A.-B., which was upregulated in metastatic melanoma tissue compared with primary melanoma tissue C.-D. 


\section{CD147 interacted with GLUT-1 in A375 cells}

In order to examine the function of CD147 and GLUT-1, we then detected the localization of these two molecules in human melanoma A375 cells (Figure 2A2B). Immunofluorescence demonstrated that CD147 and GLUT-1 was co-expressed in the cytoplasm of A375 cells in vitro culture. Plasmids expressing CD147-Myc and GLUT-1-Flag were constructed and transfected into 293FT cells. Immunoprecipitation proved that CD147 interacted with GLUT-1 (Figure 2C). Then a total of 5 CD147 deletion variants were synthesized and D34-89, D105-199, D207-230, D231-269 and D207-269 were knockdown (Figure 2D). Immunoprecipitation showed that D105-199 silencing blocked the interaction of CD147 with GLUT-1 (Figure 2E).
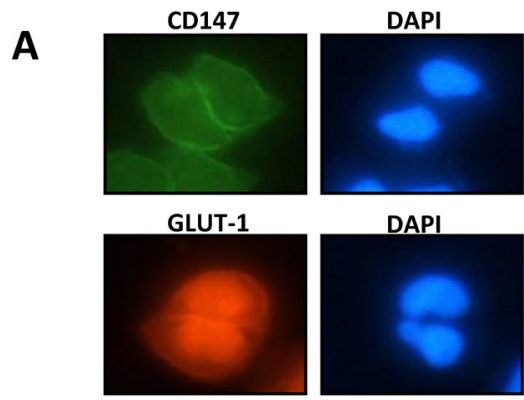

B
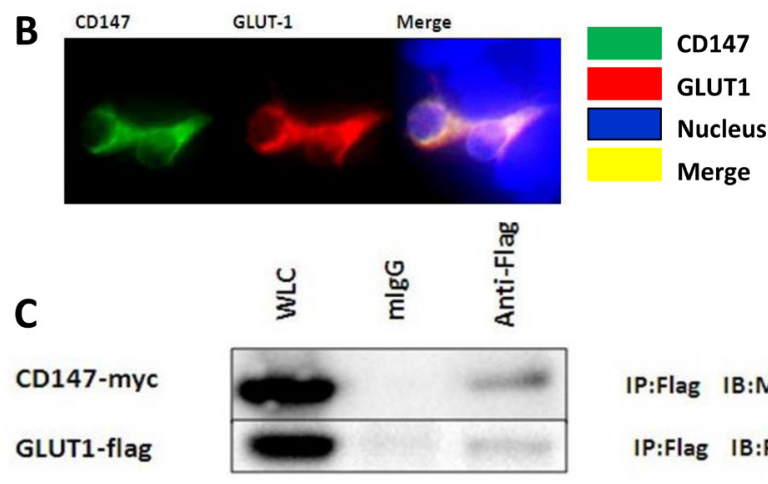

IP:Flag IB:Myc

IP:Flag IB:Flag

D

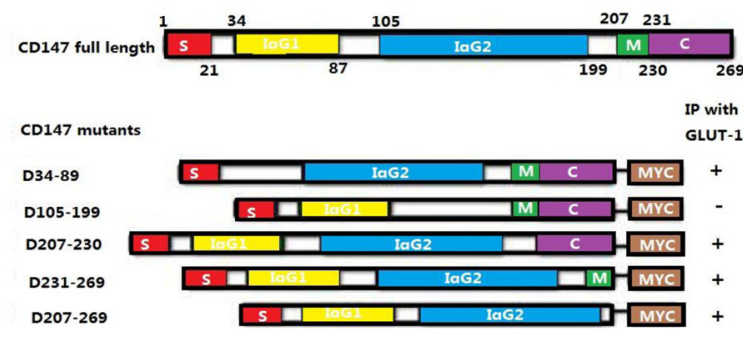

E that CD147 might regulate the expression of GLUT1. It was observed that GLUT-1 level was decreased in A375-shCD147 cells compared with that in A375 cells (Figure 3A-3C). The PI3K and p-Akt level were also downregulated by CD147 silencing (Figure 3D). LY294002, which is a specific inhibitor for PI3K/Akt signaling, could abolish the inhibitory effect of CD147 silencing on the GLUT-1 level (Figure 3E). The glucose uptake was also decreased in A375-shCD147 cells (Figure $3 F$ ), suggesting that CD147 silencing may impair the glucose supply, which is required for tumor growth in melanoma.

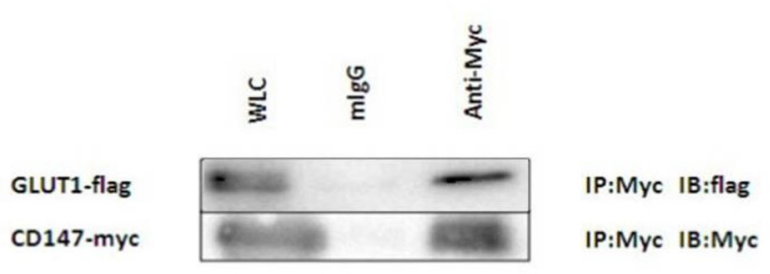
both overexpressed in melanoma tissue, we hypothesized

\section{CD147 silencing downregulated GLUT-1 via PI3K/AKT pathway}

As IHC proved that CD147 and GLUT-1 were

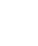
. 
Targeting CD147 could suppress tumor growth and lung metastasis in melanoma

The inhibition of CD147 silencing on melanoma was further investigated in subcutaneous mice model. Micro PET/CT scan observed that mean SUV value was $1.53 \pm 0.11$ in A375 group $(n=10)$ and $0.45 \pm 0.07$ in A375shCD147 group $(n=10)$, respectively $(P<0.05$; Figure $4 \mathrm{~A}-4 \mathrm{~B})$. IHC analysis of the tumor tissue showed that GLUT-1 level was downregulated (Figure 4C-4D). In melanoma metastasis mice model, less lung metastatic lesions were found in A375-shCD147 group comparing with those in A375 group $(P<0.05)$.

\section{DISCUSSION}

Our study for the first time reported that CD147 silencing could suppress the tumor growth by downregulating the level of GLUT-1 in melanoma. CD147 and GLUT-1 were both overexpressed in melanoma tissue and positively correlated. CD147 could modulate the expression of GLUT-1 via PI3K/Akt pathway and also interact with GLUT-1, which is an important glucose transporter.
Melanoma is highly malignant with very poor prognosis [8]. Although great effort has been made on understanding the carcinogenesis of melanoma, there are still not effective treatment $[9,10]$. Vemurafenib (Zelboraf, Roche) and dabrafenib (Tafinlar, GlaxoSmithKline) are two BRAF inhibitors, which has been applied in the treatment for melanoma [11, 12], but the survival rate remains low. Thus, it is important to identify new therapeutic targets in order to improve the clinical outcome for such patients with this deadly disease. In 1930, Warburg first found that glycolysis was common in tumor cells even under normoxia, which may be explained by the fact that tumor cells could uptake plenty of glucose and glycolysis can provide sufficient energy for maintaining the tumor growth [13]. This metabolic activity of tumor cells has attracted great attentions from researchers all over the world. Recent studies have reported that lactate dehydrogenase (LDH), pyruvate dehydrogenase (PDK) and hexokinase (HK) could serve as new therapeutic targets for treating human cancers [14]. GLUT family members facilitate the transportation of glucose into cells and GLUT-1 level was proved to be associated with oncogenic gene, growth factors, interleukin-1, local hypoxia and matrix metalloproteinases [15-17]. GLUT-1

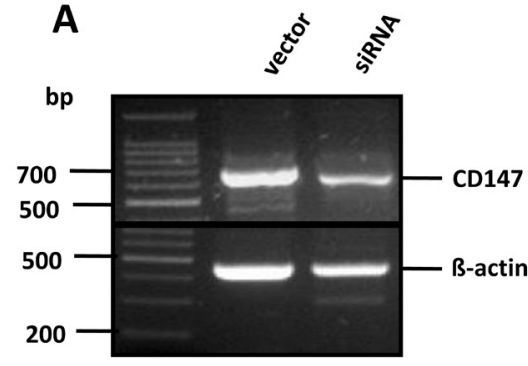

B

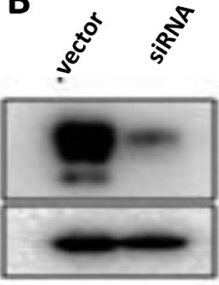

CD147

B-actin
C

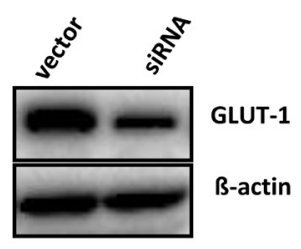

D

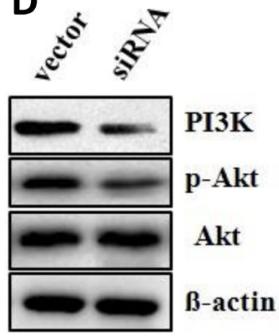

$\mathbf{E}$

$\mathbf{F}$

$\mathrm{mg} / \mathbf{1 0 5}$ cells
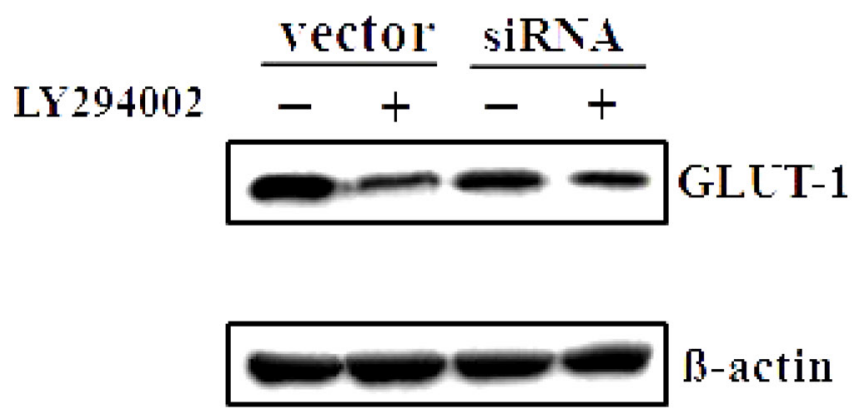

Figure 3: CD147 mRNA and protein level was decreased after transfection with CD147 siRNA in A375 cells A.-B., which lead to the downregulation of GLUT-1 via PI3K/Akt pathway C.-D. PI3K inhibitor LY294002 could abolish the inhibition of CD147 silencing on GLUT-1 E. The glucose uptake was downregulated after CD147 knockdown F. ${ }^{*} p<0.05$ compared with the vector. 
overexpression was observed in multiple human cancers, which was correlated with the invasive phenotype [1820]. Thus, impairing the function of GLUT-1 might be introduced as a new anti-tumor therapy.

In this study, we investigated the interaction of CD147 and GLUT-1. CD147 silencing could also downregulate the level of GLUT-1 and decreased glucose uptake was observed in A375-shCD147 cells. In vivo experiments also supported that CD147 silencing could inhibit the tumor growth and lung metastasis in subcutaneous mice model. PI3K/Akt signaling was involved in the regulation of CD147 on GLUT-1 in melanoma cells. Previous studies has already verified that CD147 could participate in a series of biological processes including cell proliferation, invasion and migration by inducing the production of matrix metalloproteinases [21-24]. So targeting CD147 is able to suppress the tumor growth as well as to impair the interaction between tumor cells and stroma cells. It was also proved that PI3K/Akt signaling was increased in the transformation from benign nevus to malignant melanoma [9, 25].

Taken together, our results demonstrated that CD147 knockdown could suppress the tumor growth of melanoma by downregulating GLUT-1 via PI3K/Akt pathway, which could validate CD147 silencing by specific siRNAs as a new therapeutic regimen for treating melanoma. However,

A

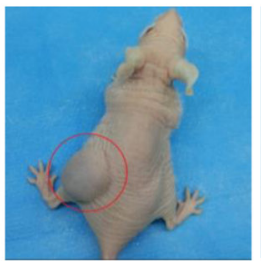

A375-shV

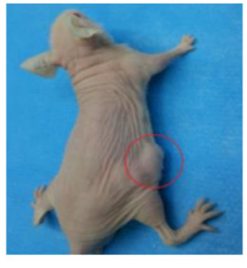

A375-shCD147
C

A375-shCD147

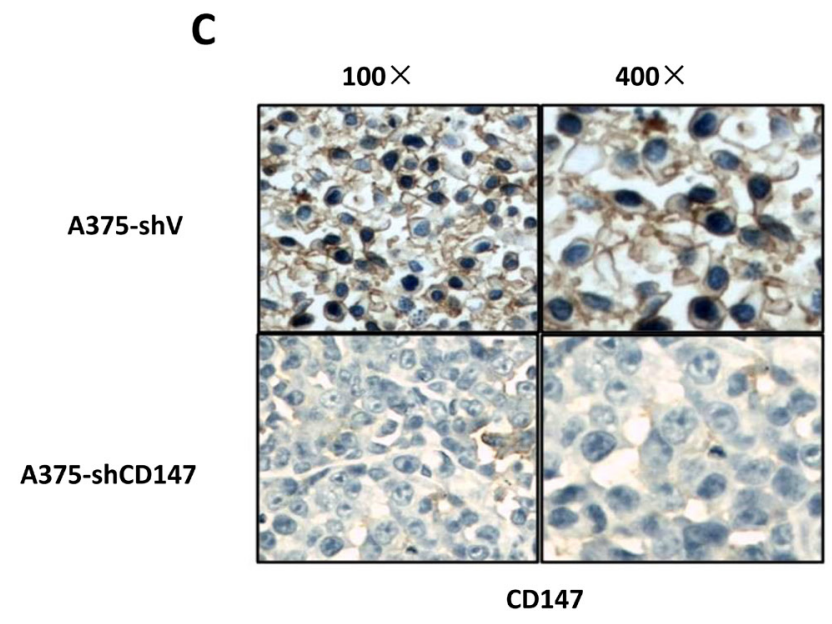

CD147 the anti-tumor action and efficacy of CD147 silencing in melanoma should be further elaborated.

\section{MATERIALS AND METHODS}

\section{Cell culture and tissue}

Human melanoma A375 cells and 293FT cells were purchased from American Type Culture Collection (ATCC). Cells were cultured in DMEM with $1 \%$ penicillin, $1 \%$ streptomycin and $10 \%$ fetal bovine serum at $5 \% \mathrm{CO}_{2}$ and $37^{\circ} \mathrm{C}$. Tissues of melanoma and pigmented nevus were collected from age- and gender- matched patients who underwent resection in our department. Our study was approved by the Ethic Committee of Zhongnan University in accordance with Declaration of Helsinki and informed consent was obtained from all the patients.

\section{Transfection and real time RT-PCR}

Specific siRNA targeting CD147 was designed and synthesized. A375 cells were transfected with CD147 siRNA and scramble siRNA (control) by lipofectamine 2000, respectively. After transfection, cells were collected
B

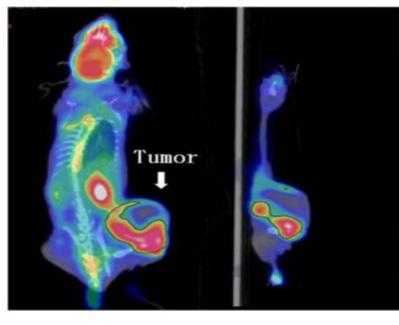

A375-shV

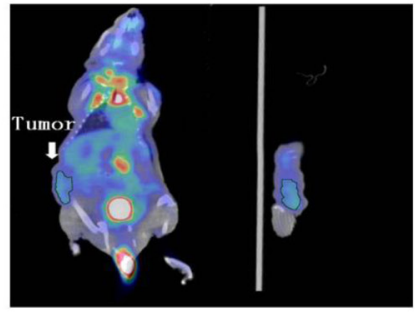

A375-shCD147
D

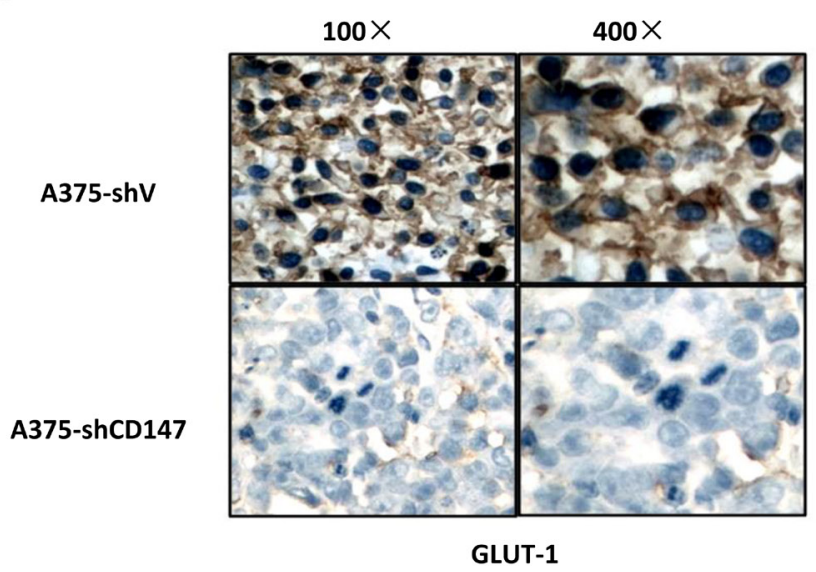

Figure 4: Decreased ${ }^{18}$ F-FDG uptake was observed by micro PET/CT in CD147 knockdown mice model A., and CD147 knockdown downregulated GLUY-1 level in mice model B. 
and RNA was extracted. Stable A375-shV and A375shCD147 cell lines were established by being transfected with constructed plasmid harboring scramble and CD147 shRNA, respectively. Real time RT-PCR was conducted to detect the mRNA level of CD147 and $\beta$-actin was used to be normalized as internal control.

\section{Western blot}

Protein sample was prepared from total cell lysate and quantified using BCA kit. 10ul protein sample was loaded in the SDS-PAGE gel for electrophoresis, which was then transferred to PVDF membrane. The blot was blocked in milk and primary antibody for detecting CD147, PI3K, p-Akt, Akt, GLUT-1 and GLUT4 were used at the dilution ratio of 1:500. $\beta$-actin served as internal control.

\section{Immunoprecipitation and immunofluorescence}

Protein $\mathrm{A}+\mathrm{G}$ agarose beads was used in the immunoprecipation to detect the interaction of CD147 and GLUT-1 and CD147 deletion variants were constructed to determine the binding site with GLUT-1. Different CD147 deletion variants with Myc tag were introduced into 293FT cells. Protein sample, cells and tissues were all first incubated in primary antibody 1:200 anti-CD147 and then secondary antibody including Cy3 1:300, FITC 1:500 and Cy5 1:300, which was observed by fluorescence microscope.

\section{Melanoma nude mice model and micro PET/CT}

A375-shV and A375-shCD147 cells were injected subcutaneously into 4-6 weeks female nude mice at the density of $10^{7} / 0.2 \mathrm{ml}$. The tumor volume was measured and recorded. After the establishment of the mice model, $0.2 \mathrm{~mL}{ }^{18} \mathrm{~F}-\mathrm{FDG}$ was injected by tail vein. Then the mice was scanned by Siemens Inveon micro PET/CT. The imaging was analyzed by Inveon Acquisition Workplace (IAW) and the region of interest (RIO) was obtained. Standard uptake value (SUV) of RIO was tested by oneway ANOVA. The melanoma metastasis mice model was also established by injecting tumor cells via tail vein. After 6 weeks, the mice were sacrificed and lung tissue was collected.

\section{Immunohistochemical analysis}

Tumor tissue was fixed in paraffin and cut into $5-\mu \mathrm{m}$ slices. 1:500 Anti-CD147 and anti-GLUT-1 antibodies were used as primary antibodies. Two pathologist evaluated all the slices independently. A total of 5 images under high power field were randomly selected for each slice. The staining could categorized into 0 (no stain), 1 (light brown), 2 (brown) and 3 (dark brown) and the positivity was classified into $0(0 \%), 1(1-25 \%), 2$ (26$50 \%)$ and $3(>50 \%)$. The averaged score (0-9) was based on the staining score $\mathrm{x}$ the positivity score, and classified into $1(0), 1(<3), 2(3-6)$ and $3(>6)$. 0 and 1 were considered as low expression and 2 and 3 were as high expression.

\section{Statistical analysis}

SPSS Statistics 17.0 software (SPSS Inc, Chicago, IL) was used for all the analysis. For categorical data, Pearson chi-square test was applied for detecting the difference. Difference on continuous data was tested by independent student $t$-test. The correlation of CD147 expression and GLUT-1 level was tested by Spearman correlation analysis. P value less than 0.05 was considered to be statistically significant.

\section{ACKNOWLEDGMENTS}

This study was supported by grants No. 81472882 from the National Natural Science Foundation of China, and 81225013 from the National Science Fund for Distinguished Young Scholars, Natural Science Foundation of Hunan (11JJ5083), The Young Talents Support Plan of Hunan, P. R. China

\section{CONFLICTS OF INTEREST}

The authors have no conflict of interest.

\section{REFERENCES}

1. Chen W, Zheng R, Baade PD, Zhang S, Zeng H, Bray F, Jemal A, Yu XQ, He J. Cancer statistics in China, 2015. CA Cancer J Clin. 2016;66:115-32.

2. Siegel RL, Miller KD, Jemal A. Cancer statistics, 2016. CA Cancer J Clin. 2016;66:7-30.

3. Kamath S, Miller KA, Cockburn MG. Current Data on Risk Factor Estimates Does Not Explain the Difference in Rates of Melanoma between Hispanics and Non-Hispanic Whites. J Skin Cancer. 2016;2016:2105250.

4. Yu L, Chen X, Wang L, Chen S. The sweet trap in tumors: aerobic glycolysis and potential targets for therapy. Oncotarget. 2016;7(25):38908-38926. doi: 10.18632/ oncotarget.7676.

5. Deng D, Yan N. GLUT, SGLT, and SWEET: Structural and mechanistic investigations of the glucose transporters. Protein Sci. 2016;25:546-58.

6. Parente P, Coli A, Massi G, Mangoni A, Fabrizi MM, Bigotti G. Immunohistochemical expression of the glucose transporters Glut-1 and Glut-3 in human malignant 
melanomas and benign melanocytic lesions. J Exp Clin Cancer Res. 2008;27:34.

7. Long T, Su J, Tang W, Luo Z, Liu S, Liu Z, Zhou H, Qi M, Zeng W, Zhang J, Chen X. A novel interaction between calcium-modulating cyclophilin ligand and Basigin regulates calcium signaling and matrix metalloproteinase activities in human melanoma cells. Cancer Letters. 2013;339:93-101.

8. Gandini S, Montella M, Ayala F, Benedetto L, Rossi CR, Vecchiato A, Corradin MT, V DEG, Queirolo P, Zannetti G, Giudice G, Borroni G, Forcignano R, et al. Sun exposure and melanoma prognostic factors. Oncol Lett. 2016;11:2706-2714.

9. Hao M, Song F, Du X, Wang G, Yang Y, Chen K, Yang J. Advances in targeted therapy for unresectable melanoma: new drugs and combinations. Cancer Letters. 2015;359:1-8.

10. Chen J, Shao R, Zhang XD, Chen C. Applications of nanotechnology for melanoma treatment, diagnosis, and theranostics. Int J Nanomedicine. 2013;8:2677-88.

11. Fawaz B, Dickson L, Menter A. Pustular psoriasis eruption with dabrafenib, a BRAF inhibitor. J Dermatolog Treat. 2016:1-4.

12. Jonas $\mathrm{O}$, Oudin MJ, Kosciuk T, Whitman M, Gertler FB, Cima MJ, Flaherty KT, Langer R. Parallel in-vivo assessment of drug phenotypes at various time points during systemic BRAF inhibition reveals tumor adaptation and altered treatment vulnerabilities. Clin Cancer Res. 2016.

13. Vander Heiden MG, Cantley LC, Thompson CB. Understanding the Warburg effect: the metabolic requirements of cell proliferation. Science. 2009;324:102933.

14. Hasanpourghadi M, Looi CY, Pandurangan AK, Sethi G, Wong WF, Mustafa MR. Phytometabolites Targeting Warburg Effect in Cancer Cells: A Mechanistic Review. Curr Drug Targets. 2016.

15. Abdou AG, Eldien MM, Elsakka D. GLUT-1 Expression in Cutaneous Basal and Squamous Cell Carcinomas. Int J Surg Pathol. 2015;23:447-53.

16. Gunnink LK, Alabi OD, Kuiper BD, Gunnink SM, Schuiteman SJ, Strohbehn LE, Hamilton KE, Wrobel KE, Louters LL. Curcumin directly inhibits the transport activity of GLUT1. Biochimie. 2016;125:179-85. doi: 10.1016/j. biochi.2016.03.014.

17. Zhao F, Ming J, Zhou Y, Fan L. Inhibition of Glut1 by WZB117 sensitizes radioresistant breast cancer cells to irradiation. Cancer Chemother Pharmacol. 2016;77:963-72.

18. Venturelli L, Nappini S, Bulfoni M, Gianfranceschi G, Dal Zilio S, Coceano G, Del Ben F, Turetta M, Scoles G, Vaccari L, Cesselli D, Cojoc D. Glucose is a key driver for GLUT1-mediated nanoparticles internalization in breast cancer cells. Sci Rep. 2016;6:21629.

19. Fan JY, Yang Y, Xie JY, Lu YL, Shi K, Huang YQ. MicroRNA-144 mediates metabolic shift in ovarian cancer cells by directly targeting Glut1. Tumour Biol. 2016;37:6855-60.

20. Stewart PA, Parapatics K, Welsh EA, Muller AC, Cao H, Fang B, Koomen JM, Eschrich SA, Bennett KL, Haura EB. A Pilot Proteogenomic Study with Data Integration Identifies MCT1 and GLUT1 as Prognostic Markers in Lung Adenocarcinoma. PLoS One. 2015;10:e0142162.

21. Fu ZG, Wang L, Cui HY, Peng JL, Wang SJ, Geng JJ, Liu JD, Feng F, Song F, Li L, Zhu P, Jiang JL, Chen ZN. A novel small-molecule compound targeting CD147 inhibits the motility and invasion of hepatocellular carcinoma cells. Oncotarget. 2016;7:9429-47. doi:10.18632/oncotarget.6990.

22. Liu H, Xu XF, Zhao Y, Tang MC, Zhou YQ, Gao FH. NS398 promotes pancreatic cancer cell invasion by CD147 and MMP2 via the activation of P38. Mol Med Rep. 2016;13:2208-14.

23. Cui HY, Wang SJ, Miao JY, Fu ZG, Feng F, Wu J, Yang XM, Chen ZN, Jiang JL. CD147 regulates cancer migration via direct interaction with Annexin A2 and DOCK3-betacatenin-WAVE2 signaling. Oncotarget. 2016;7:5613-29. doi:10.18632/oncotarget.6723.

24. Dai L, Trillo-Tinoco J, Chen Y, Bonstaff K, Del Valle L, Parsons C, Ochoa AC, Zabaleta J, Toole BP, Qin Z. CD147 and downstream ADAMTSs promote the tumorigenicity of Kaposi's sarcoma-associated herpesvirus infected endothelial cells. Oncotarget. 2016;7:3806-18. doi:10.18632/oncotarget.6584.

25. Cohen-Solal KA, Boregowda RK, Lasfar A. RUNX2 and the PI3K/AKT axis reciprocal activation as a driving force for tumor progression. Mol Cancer. 2015;14:137. 